\title{
Conditioned approach-withdrawal behavior and some signal-food relations in pigeons: Performance and positive vs. negative "associative strength"
}

\author{
ELIOT HEARST, SARAH W. BOTTJER, and EDWARD WALKER \\ Indiana University, Bloomington, Indiana 47405
}

\begin{abstract}
The location of pigeons was monitored during illuminations of a key (CS) presented in a positive, zero, or negative relationship with food for different groups of subjects. Birds approached a signal positively correlated with food and withdrew from a negatively correlated signal. When the probability of food in the absence of CS was increased from 0 to .2 while the probability of food during CS remained constant at .4, approach to CS declined. When the probability of food during CS increased from 0 to .2 while the probability of food in CS absence remained constant at .4, withdrawal from CS declined. No consistent approach or withdrawal appeared when the probability of food was the same (.4) during the presence and absence of CS. Our approach-withdrawal assay of positive and negative associative strength, which allows trial-by-trial measurement of overt performance during excitatory or inhibitory conditioning, was used to assess several predictions of the Rescorla-Wagner model of Pavlovian conditioning.
\end{abstract}

Recent influential theories of Pavlovian conditioning have attempted to encompass both excitatory and inhibitory processes in their formulations. Rescorla's (1967) contingency approach proposed that the relative rate of unconditioned stimulus (US) occurrence in the presence vs. absence of the conditioned stimulus (CS) determines whether the CS will become excitatory or inhibitory: When USs occur more often in CS than in the absence of CS (to be denoted $\overline{C S}$ ), excitatory conditioning develops to CS, and when USs are more likely in $\overline{\mathrm{CS}}$ than in CS, inhibitory conditioning develops to CS. On the other hand, when USs are equally likely in CS and $\overline{\mathrm{CS}}$, Rescorla argued that CS provides no information about occurrences of US, despite many "pairings" of CS and US, and that therefore no conditioning to CS should take place.

The more contiguity-based, molecular formulation that was subsequently proposed by Rescorla and Wagner (1972; Wagner \& Rescorla, 1972) stressed the role of background stimulation and provided a relatively explicit model for making predictions about the growth of positive and negative associative strength. In several places (e.g., Rescorla, 1972, p. 20; Rescorla \& Wagner, 1972, p. 89), computer simulations of the model have been presented that indicate how excitation and inhibition should develop over trials of acquisition when the

This research was supported by National Institute of Mental Health Grant MH19300. We thank Alida Evans and Dexter Gormley for valuable advice and assistance. Sarah Bottjer is now at the Department of Psychology, University of California at Los Angeles. Requests for reprints should be sent to Eliot Hearst, Department of Psychology, Indiana University, Bloomington, Indiana 47405. probability of a US in CS is held constant and its probability in $\overline{\mathrm{CS}}$ is varied, and vice versa. Unfortunately, however, most empirical tests of these predictions have been forced to rely on relatively brief postconditioning assays, such as summation or retardation tests (Rescorla, 1969; Witcher \& Ayres, 1980). Measurement of trialby-trial behavioral changes during original conditioning, which might permit the mapping of associative strength into performance, has not been feasible with the tasks employed, particularly in the case of inhibitory learning.

In the present experiment, a sign-tracking arrangement (Hearst \& Jenkins, 1974; Wasserman, Franklin, \& Hearst, 1974) was used to allow measurement of the growth of approach or withdrawal responses with respect to signals positively or negatively correlated with food, respectively; these behaviors were recorded from the very first trial of conditioning and until asymptotic performance had been approximately reached. The basic method, initially developed by Wasserman et al., involved a situation in which the CS (illumination of a single key) is presented to pigeons in two possible locations (left or right) on one wall of a standard chamber, and the pigeon's movements toward or away from the CS are recorded by means of a teeter-totter floor. We employed this procedure here to determine whether various features of the development of positive and negative associative strength that were derived from simulations of the Rescorla-Wagner model would be reflected in the actual approach-withdrawal behavior of pigeons. Thus we held constant the probability of US occurrence in CS and varied the probability of US occurrence in $\overline{\mathrm{CS}}$ for some of the groups, and we performed the reverse manipulation in other groups. 
Although Hearst and Franklin (1977, Experiment 3) explored the effects of a range of positive and negative relations between CS and US on approach-withdrawal behavior in a very similar paradigm, they did not hold constant one of these two critical probabilities while varying the other. Therefore, their results did not provide data that are directly relevant for evaluating specific predictions from the simulations presented by Rescorla and Wagner.

To check on whether the approach-withdrawal index that we continuously monitored during original conditioning would yield conclusions like those obtained from more conventional retardation-of-excitatoryconditioning tests, and to evaluate further the validity of approach-withdrawal behaviors for empirically mapping positive and negative associative strength, at the end of the experiment we placed all subjects on a highly positive CS-US contingency and examined their early performance and relative rates of acquiring strong directed movements toward the CS.

\section{METHOD}

\section{Subjects}

Forty-two experimentally naive female White Carneaux pigeons, 5-7 years old and maintained at $75 \%$ of their freefeeding weights, served as subjects.

\section{Apparatus}

Four standard pigeon chambers were used. Either the left or the right key $(18 \mathrm{~cm}$ apart) was illuminated with white light on a given trial by means of a miniprojector mounted behind each key. A houselight was centered above and midway between the two keys and remained on throughout all experimental sessions. A 3-sec presentation of grain served as the reinforcer (US). A teeter-totter floor detected whether a bird was standing on the right or left half of the chamber, that is, near the right or left key, respectively.

\section{Procedure}

Many aspects of the general method were the same as those described in articles by Hearst and Franklin (1977) and Wasserman et al. (1974), which should be consuited about details not covered in the present report.

After standard magazine training, subjects received 16 daily sessions of exposure to certain Pavlovian contingencies between key illumination (CS) and food delivery. The specific relation between CS and US was varied for the seven different groups, to which the subjects were randomly assigned, but for all subjects each session consisted of an approximately equal number of 12 -sec illuminations of the left or the right key. The sequence of left and right key illuminations was nonsystematic, with the constraint that no more than three consecutive trials could involve illumination of the same key. Each bird received an assortment of different sequences over successive experimental days. The keys remained dark during intertrial intervals, which averaged $48 \mathrm{sec}$ (range $=24-72 \mathrm{sec}$ ).

All sessions were divided into successive 3-sec "probability units," and gating devices were used to control probabilities of grain delivery during each 3 -sec period that the CS was present or absent. Grain delivery immediately occurred whenever a positive output was emitted from the gating circuit, which was sampled at the start of every 3 -sec period in the session. Experimental Groups .4-0, .4-.2, .4-.4, .2-.4, and 0-.4 $(n=7$ in the first four groups, and $n=6$ in the last group) received a total of
$30 \mathrm{CSs} / \mathrm{session}$, with the first number of the group's label (see Rescorla \& Wagner, 1972) referring to the probability of US delivery during any (12-sec) CS and the second number to the probability of US delivery during any 12 -sec period ( $\overline{\mathrm{CS}}$ ) when CS was absent; $\overline{C S}$ constituted $80 \%$ of the total session, as indicated above. Sessions lasted $30 \mathrm{~min}$ for these five groups. Therefore, on each day, Group .4-0 was scheduled to receive 12 USs in CS and 0 USs in CS; Group .4-.2, 12 USs in CS and 24 in $\overline{\mathrm{CS}}$; Group .4-.4, 12 USs in CS and 48 in $\overline{\mathrm{CS}}$; Group .2-.4, 6 USs in CS and 48 in $\overline{\mathrm{CS}}$; and Group 0-.4, 0 USs in CS and 48 in $\overline{\mathrm{CS}}$.

Because the total number of reinforcements per session varied for the above groups, a difference that was especially large for the $.4-0$ and $.4-.2$ subjects compared with the others, two additional comparison groups $(n=4)$ were also included in the experiment. These subjects were exposed to the same CS-US contingencies as were the previously described $.4-0$ and $.4-.2$ groups, but the length of their sessions was extended to 135 and $45 \mathrm{~min}$, respectively. Both of these additional groups thus received a total of 54 food deliveries, a number within the range of USs obtained daily by the $.4-.4, .2-.4$, and $0-.4$ groups. In addition to providing a control for total number of daily USs compared with the other groups, these two groups also allowed assessment of possible differences in asymptotic behavioral levels that depended on the number of USs given per day on the .4-0 and .4-.2 contingencies themselves.

Use of the probability gates was successful in producing approximately the scheduled number of USs in CS and $\overline{C S}$. The actually obtained mean probabilities of a US in 12-sec periods of CS and $\overline{C S}$ over the 16 days of training were $.37-0, .36-18$, $.36-.36, .19-.36$, and $0-.36$ in the main .4-0, .4-.2, .4-.4, .2-.4, and $0-.4$ groups, respectively, and $.37-0$ and $.39-18$ in the prolonged-session $.4-0$ and $.4-.2$ groups, respectively. Because the nominal and actual values were so close, we will refer to the groups according to the labels assigned to them before the experiment.

After 16 sessions of exposure to the above treatments, all subjects in the seven groups received 14 daily 30 -min sessions on the .4-0 contingency. This procedure corresponds to the postconditioning retardation-of-learning test recommended by Rescorla (1969) to detect the effects of prior exposure to various CS-US contingencies.

Our measure of the behavioral control exerted by CS in the various groups was the approach-withdrawal index used by Hearst and Franklin (1977) and Wasserman et al. (1974) and referred to by Hearst and Jenkins (1974) in terms of "signtracking." We recorded the cumulative amount of time during key illuminations that the subject stood on the same side of the chamber as the lighted key. An approach-withdrawal ratio was derived from these data and indicated whether the bird positioned itself near or far from the lighted key. The ratio was calculated by the formula: total time spent on the same side as the CS divided by total time that the CS was on. A ratio of .50 would indicate that the bird's movements were not systematically controlled by the location of the key light. Ratios near .0 or 1.0 would indicate strong withdrawal or approach to CS, respectively.

Data concerning approach times were recorded on every trial for all subjects during the 16 sessions of initial training, but only the group data are analyzed in this report.

\section{RESULTS}

Mean approach-withdrawal ratios are presented in Figure 1 for successive two-session blocks of training and retardation testing in each of the seven groups. Subjects exposed to positive contingencies between CS and US (.4-0 and .4-.2) developed consistent approach 


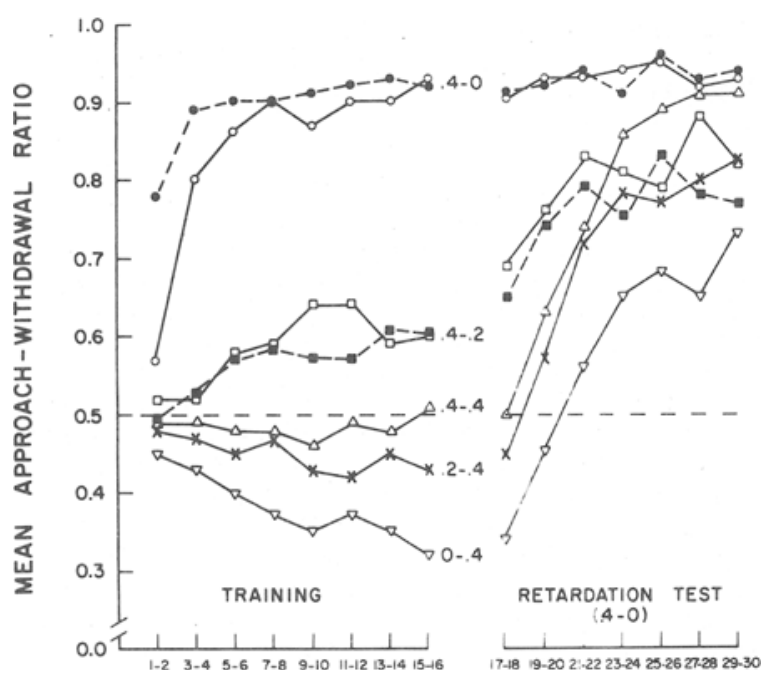

SUCCESSIVE SESSION BLOCKS

Figure 1. Approach-withdrawal behavior during CS for groups exposed to different Pavlovian contingencies between CS and US in the training phase (Sessions 1-16) and then all placed on the .4-0 contingency during the final 14 sessions (retardation test). The first number of each group label refers to the probability of US occurrence during CSs, and the second number refers to the probability of US occurrence during equivalent non-CS periods. The dashed-line curves for the .4-0 and .4-.2 treatments display data for the control groups given longer sessions than the other five groups (solid lines), so that the total daily number of USs for the former two groups would fall within the range of total USs received by subjects in the $.4-.4, .2-.4$, and 0-.4 groups.

behavior toward CS during the training phase, although the stronger positive contingency (.4-0) produced substantially higher approach levels than did the weaker contingency (.4-.2). The zero contingency (.4-.4) yielded no consistent evidence of approach or withdrawal, but subjects exposed to negative contingencies between CS and US (.2-.4 and 0-.4) developed consistent withdrawal behavior from CS, with the stronger negative contingency producing stronger withdrawal. Subjects in the .4-0 and .4-.2 groups that received 54 USs/session, as a result of being exposed to sessions that lasted 4.5 and 1.5 times longer than their respective counterparts, reached performance asymptotes that were approximately the same as their counterparts; however, asymptotic behavior was attained more rapidly in the .4-0 group by subjects receiving 135 daily trials (dashed lines in Figure 1) than by those receiving only 30 daily trials (solid lines).

Statistical analysis supported the general conclusions derived from visual inspection of the Figure 1 training data. Asymptotic performance (calculated from median scores of individual subjects over the last three session blocks, i.e., Sessions 11-16) was significantly greater than the .50 baseline for Groups $.4-0$ and $.4-.2$ receiving 30 daily trials $[\mathrm{t}(6)=10.53, \mathrm{p}<.001$, and $\mathrm{t}(6)=3.61$, $\mathrm{p}<.02$, respectively] , and for the $.4-0$ and $.4-.2$ groups receiving longer sessions $[\mathrm{t}(3)=15.94, \mathrm{p}<.001$, and $t(3)=21.95, p<.001$, respectively $]$. On the other hand, asymptotic performance in Group .4-.4 did not differ significantly from the .50 baseline $[\mathrm{t}(6)=.99]$. However, asymptotic performance was significantly lower than the .50 baseline for Groups $.2-.4$ and $0-.4$ $[\mathrm{t}(6)=4.82, \mathrm{p}<.01$, and $\mathrm{t}(5)=5.08, \mathrm{p}<.01$, respectively].

When the asymptotic performance of each of the seven groups was compared with that of every other group by means of individual $t$ tests, all differences between them were significant beyond the .05 level, except for the .4-.4 vs. .2-.4 comparison, which just missed the .05 level $[t(12)=2.07]$. Thus it seems clear that the positive contingencies tested in this experiment both produced highly significant approach behavior toward CS; the zero contingency (Rescorla's, 1967, "truly random control") yielded no systematic approach or withdrawal behavior with respect to CS; and the negative contingencies produced withdrawal from CS.

After the 16 sessions of acquisition, all subjects were exposed to the 4-0 contingency for 14 sessions. The right-hand panel of Figure 1 shows that approach behavior subsequently developed in all three groups that had not received prior training on a positive contingency; and approach behavior reached higher levels than before in the group (.4-.2) trained previously with a weaker positive contingency. Measures of approach-withdrawal remained perfectly ordered for three session blocks (Sessions 17-22) with respect to their values at the end of training, but during Sessions 23-30 overlap among the groups began to occur. By the end of retardation testing, it was not yet clear whether all groups would ever attain the performance levels achieved by subjects that had continued on the .4-0 contingency from the very start of the experiment.

\section{DISCUSSION}

Use of approach-withdrawal behavior as an assay of positive or negative associative strength yielded acquisition curves that support many of the predictions derived from simulations of the Rescorla-Wagner model (e.g., Rescorla, 1972, p. 20; Rescorla \& Wagner, 1972, p. 89). As assumed in those simulations, the CS was present $20 \%$ of the time in our work, too. The results exhibited a clear ordering of groups according to the relative probabilities of US occurrence in the presence and absence of CS; approach-withdrawal ratios significantly above the .50 baseline were obtained with positive contingencies between the CS and food, and ratios significantly below .50 were obtained with negative contingencies. Stronger positive or negative correlations produced stronger approach or withdrawal, respectively. Furthermore, when the probability of US occurrence in equivalent $\mathrm{CS}$ and $\overline{\mathrm{CS}}$ periods was the same, no evidence of approach or withdrawal with respect to CS was observed.

Effects revealed during the initial blocks of the postconditioning retardation-of-excitatory-learning phase confirm the above general conclusions and suggest that use of the approachwithdrawal measure during original acquisition provides a valid way of obtaining trial-by-trial data, enabling (1) the possible mapping of associative strength into performance during successive trials or stages of original conditioning and (2) parameter estimation in precise quantitative assessments of the RescorlaWagner model. Worth mentioning in connection with these points is the observation that organisms apparently will display 
a reversal of the approach-withdrawal behavior obtained here if aversive USs are employed: They tend to withdraw from CSs positively correlated with shock and to approach safety signals (see Hearst \& Jenkins, 1974, pp. 43-44; LeClerc \& Reberg, in press). Thus CS approach-withdrawal indices may also prove valuable in securing detailed acquisition data with the kind of US used in most prior assessments of the Rescorla-Wagner model (for example, noxious stimulation in eyelid conditioning and CER procedures).

There are at least two types of predictions from the model that are not clearly confirmed by the present set of findings. First, the model predicts that asymptotic performance on a given treatment will not be affected by prior contingencies to which the organism has been exposed. The final sessions of the retardation test (for example, Sessions 27-30 in Figure 1) did not reveal overall attainment of the levels achieved by the group that had been on the .4-0 treatment throughout the entire experiment. However, one could argue that behavior had not yet reached asymptote in several groups by Sessions 27-30 and, if continued longer in the experiment, all groups would eventually have exhibited approach-withdrawal ratios of approximately .90 .

Second, the simulations of the model presented by Rescorla (1972, p. 20) and Rescorla and Wagner (1972, p. 89) yielded curves that were frequently nonmonotonic in shape; associative strengths under certain positive, zero, and negative CS-US contingencies often showed an initial rise followed by a decline to their asymptotic values. Examination of this interesting prediction about preasymptotic effects was one of our main reasons for performing the present experiment. Clearly, the data of Figure 1 reveal no evidence of such nonmonotonic learning curves. If such effects do exist, our failure to detect them could have been due to the particular overall US density, CS salience, or US magnitude we employed; Rescorla and Wagner (1972) have discussed how manipulation of these and other factors should affect the amount and duration of the initial positive changes in CS associative strength that presumably often precede later decreases. Furthermore, because of averaging, our use of group data may have obscured initial rises and subsequent declines in approach-withdrawal behavior that occurred in individual subjects. However, visual inspection of trial-by-trial acquisition curves for approximately half of the individuals in the experiment failed to reveal any definite indications of the nonmonotonic functions that should occur under conditions like ours, according to the Rescorla-Wagner model. In view of the fact that retardation and summation tests following aversive conditioning treatments have supported the existence of such nonmonotonicities (see Rescorla, 1972), a critic might question the sensitivity of our paradigm for detecting those kinds of effects. Obviously, further variation of CS and US density and magnitude or salience in the present situation would be useful in evaluation of this point and other predictions of the model.

In general, measures of approach-withdrawal provided performance indices that confirmed the main predictions of the model with respect to simple Pavlovian conditioning and seem quite relevant to its further analysis. In our arrangement, retardation of excitatory conditioning by previous exposure to negative correlations between CS and US cannot easily be attributed to possible habituation or loss of attention to the CS by the subject during original training (see Rescorla, 1969), since it seems logically impossible for subjects to consistently move away from a stimulus to which they have habituated or to which they do not attend. The greater "shallowness" (magnitude) of the inhibitory effects below the .50 baseline in Figure 1, compared with the excitatory effects above the .50 baseline, may result from the fact that subjects can "withdraw" from a stimulus not only by shuttling to or remaining on the other side of the chamber, but also by moving backward or turning around on the same side. (Informal observations of birds exposed to negative contingencies in our laboratory have frequently revealed evidence of such behaviors.) Effects of this kind might produce underestimation of inhibitory strength and would make our measure of "inhibition" less sensitive in certain respects than our measure of "excitation," which always entails directed approach toward a particular object in space (cf. Witcher \& Ayres, 1980, pp. 72-73). Nevertheless, the fact that a definite response is produced by a so-called "conditioned inhibitor" in our paradigm and that findings based on measures of this response seem to correlate highly with results of postconditioning summation and retardation assays in various other studies seem valuable points to consider in planning future empirical and theoretical research on Pavlovian conditioning.

\section{REFERENCES}

Hearst, E., \& Franklin, S. R. Positive and negative relations between a signal and food: Approach-withdrawal behavior to the signal. Journal of Experimental Psychology: Animal Behavior Processes, 1977, 3, 37-52.

Hearst, E., \& Jenkins, H. M. Sign-tracking: The stimulusreinforcer relation and directed action. Austin, Tex: Psychonomic Society, 1974.

LeCle RC, R., \& Reberg, D. Sign-tracking in aversive conditioning. Learning and Motivation, in press.

Rescorla, R. A. Pavlovian conditioning and its proper control procedures. Psychological Review, 1967, 74, 71-80.

Rescorla, R. A. Pavlovian conditioned inhibition. Psychological Bulletin, 1969, 72, 77-94.

RESCorla, R. A. Informational variables in Pavlovian conditioning. In G. H. Bower (Ed.), The psychology of learning and motivation (Vol. 6). New York: Academic Press, 1972.

Rescorla, R. A., \& Wagner, A. R. A theory of Pavlovian conditioning: Variations in the effectiveness of reinforcement and nonreinforcement. In A. H. Black \& W. F. Prokasy (Eds.), Classical conditioning II: Current research and theory. New York: Appleton-Century-Crofts, 1972.

Wagner, A. R., \& Rescorla, R. A. Inhibition in Pavlovian conditioning: Application of a theory. In R. A. Boakes \& M. S. Halliday (Eds.), Inhibition and learning. New York: Academic Press, 1972.

Wasserman, E. A., Franklin, S. R., \& Hearst, E. Pavlovian appetitive contingencies and approach versus withdrawal to conditioned stimuli in pigeons. Journal of Comparative and Physiological Psychology, 1974, 86, 616-627.

Witcher, E. S., \& Ayres, J. B. Systematic manipulation of CS-US pairings in negative CS-US correlation procedures in rats. Animal Learning \& Behavior, 1980, 8, 67-74.

(Received for publication June 21, 1980.) 